\title{
STRUCTURE IN SIMPLEXES. III. COMPOSITION SERIES $\left({ }^{1}\right)$
}

\author{
BY \\ EDWARD G. EFFROS AND ALAN GLEIT
}

The Jacobson structure space has played a central role in the algebraic classification of $C^{*}$-algebras. Kaplansky showed [9] that for $G C R$ algebras, one may find an ascending transfinite "composition series" of open sets $G_{\alpha}$ exhausting the structure space, such that $G_{\alpha+1}-G_{\alpha}$ is Hausdorff, and the component algebra over $G_{\alpha+1}-G_{\alpha}$ is homogeneous. The latter algebras may be characterized as continuous sections of certain fiber bundles (see [6, §10]); hence the classification of $G C R$ algebras is reduced to finding how homogeneous algebras may be "put together".

An analogous structure space was defined for Choquet simplexes, and more generally for simplex spaces in [7] and [8]. In this paper we shall investigate composition series for simplex spaces. The most promising candidates for the components of such a composition series are the " $C$-spaces", the simplex spaces of the form $C_{0}(X)$, where $X$ is a locally compact Hausdorff space. We say that a simplex space is a " $G C$-space" if it has such a composition series. We have succeeded in characterizing the separable $G C$-spaces in Theorem 5.1 as the separable simplex spaces for which the extremal states form an $F_{\sigma}$ subset of the states, and closed subsets of the structure space are second category in themselves. By constructing various $M$-spaces in $\S 7$, we show that both of these conditions are necessary, even for this restricted class of simplex spaces.

$\$ 1$ is devoted to several preliminary results concerning closed ideals and quotients of simplex spaces. In $\$ 2$ we define a " $C$-ideal" in a simplex space to be a closed ideal which is itself a $C$-space. In Theorem 2.2 we show that if $J$ is such an ideal in a simplex space $A$, then points in $\max J$ may be separated from distinct points in $\max A$ by disjoint open sets. This and other results are false (see $\$ 7.1$ ) when one assumes only that $J$ is an $M$-space, or equivalently (see Corollary 2.6) that max $J$ is Hausdorff. To avoid this difficulty, we define an " $M$-ideal" $J$ to be a closed ideal for which $\max J$ is "separated" in $\max A$. Equivalent conditions are given in Theorem 2.5.

In $\$ \S 3$ and 4 we consider a general theory of composition series. The reader may wish to avoid the somewhat tedious and routine proofs of these sections on a first reading of the paper. The theory is applicable when the components are $C$-ideals, $M$-ideals, or $M$-ideals with locally compact structure. The simplex spaces which

Received by the editors March 15, 1968 and, in revised form, November 1, 1968. 7683).

(1) This research was supported in part by the National Science Foundation (NSF GP- 
are exhausted by such series are called $G C$-, $G M$-, and $G M L C$-spaces, respectively. These are distinct classes even for the separable $M$-spaces (see $\$ 7$ ).

In $\$ 5$ we prove the main theorems of the paper. Separable $G C$ - and $G M L C$ spaces are characterized in Theorems 5.1 and 5.2. Necessary and sufficient conditions for a $G M$-space to be $G M L C$ are given in Theorem 5.3.

In $\$ 6$ we give a simplex space analogue of the Bishop-de Leeuw peaking description of the Choquet boundary of a function algebra. This in part generalizes a result of Rogalski for simplex spaces with "good spectrum" (see [15, Theorem 3]). We conclude by constructing various examples in $\$ 7$.

We shall use the notation and definitions of [7] and [8]. We always assume that a topological space is nonempty, and that a simplex space has at least one nonzero vector. We recall that if $A$ is a simplex space, there is a continuous one-to-one map of $E P_{1}(A)-\{0\}$ onto $\max A$ defined by $p \rightarrow\{p\}^{\perp}$. We will write $M_{A}(p)$ or $M_{p}$ for the latter ideal, and $p(M)$ or $p_{M}$ for the pre-image of a maximal ideal $M$. Regarding the elements of $A$ as functions on both $E P_{1}(A)-\{0\}$ and $\max A$, we have $a(M)=a\left(p_{M}\right)$. These functions define the topology on $E P_{1}(A)-\{0\}$. The closure of $E P_{1}(A)$ is denoted by $Z_{A}$.

If $Q$ is a closed face containing 0 in $P_{1}(A)$, we let

$$
R(Q)=\{\alpha p: p \in E(Q), 0 \leqq \alpha \leqq 1\}=[0,1] E(Q),
$$

i.e., $R(Q)$ consists of the "extremal rays" in $Q$.

By an isomorphism of simplex spaces, we shall mean an isometric order isomorphism.

1. Ideals and quotients. Let $I \neq A$ be a closed ideal in a simplex space $A$, and $\theta: A \rightarrow A / I$ be the quotient map. Then the adjoint map $\theta^{*}:(A / I)^{*} \rightarrow A^{*}$ is an isometry and a weak* homeomorphism onto $I^{0}$. As we saw in [7, Theorem 3.4], $\theta^{*}$ is also an order isomorphism; hence it restricts to an affine isomorphism of $P_{1}(A / I)$ onto the face $I^{\perp}$ in $P_{1}(A)$, and thence to a homeomorphism of $E P_{1}(A / I)-\{0\}$ onto $E\left(I^{\perp}\right)-\{0\}$. From [7, Theorem 4.4], if $M \in \max A / I$, then $\theta^{-1}(M) \in \max A$ and $M \rightarrow \theta^{-1}(M)$ is a homeomorphism of $\max A / I$ onto $h(I)$. Furthermore the diagram

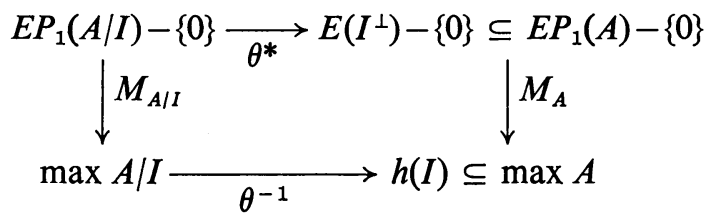

is commutative. We shall often identify $\max A / I$ and $h(I)$ as topological spaces. If we regard the elements of $A / I$ as functions on $\max A / I$, they may be identified with the restrictions of elements of $A$ to the closed set $h(I)$.

Let $J \neq\{0\}$ be a closed ideal in a simplex space $A$. From [7, Theorem 4.4], the restriction map $\rho: A^{*} \rightarrow J^{*}$ restricts to a continuous affine map $\rho_{1}$ of $P_{1}(A)$ onto 
$P_{1}(J)$, and thence to a continuous one-to-one map $\rho_{E}$ of $E P_{1}(A)-J^{\perp}$ onto $E P_{1}(J)-\{0\}$. If $M \in \max A-h(J)$, then $\eta_{A}(M)=M \cap J \in \max J$, and $\eta_{A}$ is a homeomorphism of $\max A-h(J)$ onto $\max J$. The diagram

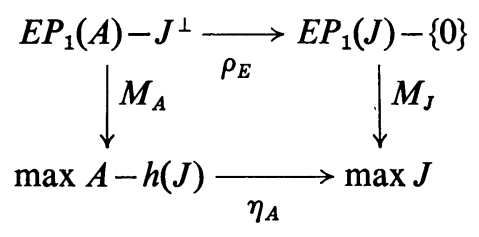

is commutative. We shall often identify $\max J$ and $\max A-h(J)$ as topological spaces. If we regard elements of $J$ as functions on $\max J$, then they may be identified with the elements in $A$ which vanish off the open set $\max A-h(J)$.

It is important to note that $\rho_{E}$ apparently need not be a homeomorphism; hence the elements of $J$ might not determine the topology on $E P_{1}(A)-J^{\perp}$. On the other hand we do have

LEMMA 1.1. If $K$ is a compact subset of $E P_{1}(J)-\{0\}$, then $\rho_{E}^{-1}(K)$ is compact in $E P_{1}(A)-J^{\perp}$.

Proof. Letting $\rho_{1}: P_{1}(A) \rightarrow P_{1}(J)$ be the restriction map, it suffices to show that

$$
\rho_{1}^{-1}\left(E P_{1}(J)-\{0\}\right) \subseteq E P_{1}(A)-J^{\perp}
$$

since then $\rho_{E}^{-1}(K)=\rho_{1}^{-1}(K)$ will be closed and thus compact in $P_{1}(A)$.

If $q \in E P_{1}(J)-\{0\}$, the set $Q=\rho_{1}^{-1}(q)$ is a closed nonempty face in $P_{1}(A)$ disjoint from the face $J^{\perp}$. If $Q$ did not consist of a single extreme point, $E(Q)$ would contain more than one point. Since $E(Q) \subseteq E P_{1}(A)-J^{\perp}$, this would contradict the fact that $\rho_{1}$ is one-to-one on the latter set. Thus $\rho_{1}^{-1}(q)=\{p\}$, where $p \in E P_{1}(A)-J$.

If $I$ and $J$ are closed ideals in a simplex space $A$, then Davies has shown [4] that $J+I$ is again a closed ideal. We also have

LEMMA 1.2. Suppose that $I \neq A, J \neq\{0\}$, and that $\theta_{I}$ is the quotient map of $A$ onto $A / I$. Then the relative and quotient norms and orderings coincide on $\theta_{I}(J)=J+I / I$, and the latter is a closed ideal in $A / I$. Let us identify $\max J+I / I$ with an open subset of $\max A / I$, and then identify $\max A / I$ and $\max J$ with closed and open subsets of $\max A$, respectively. When regarded as subsets of $\max A$,

$$
\max J+I / I=\max J \cap \max A / I \text {. }
$$

Proof. The remark concerning norms is trivial. The relative ordering is defined by the cone

$$
\begin{aligned}
J+I / I \cap(A \mid I)^{+} & =J+I / I \cap A^{+} / I \\
& =(J+I) \cap A^{+} / I=(J+I)^{+} / I,
\end{aligned}
$$


and the latter is the quotient cone. Since $J+I / I$ is a simplex space [7, Theorem 3.4], it follows that $J+I / I$ is closed in $A / I$, and positively generated. It is an ideal, for suppose that

$$
0 \leqq a+I \leqq b+I, \quad b+I \in J+I / I .
$$

Then letting $u, v \in I$ be such that $0 \leqq a+u, a \leqq b+v$, we have

$$
0 \leqq a+u \leqq b+u+v \in J+I,
$$

hence $a+u \in J+I$, and $a+I \in J+I / I$.

We have

$$
\begin{aligned}
\max J+I / I & =\{M \in \max A / I: M \ddagger J+I / I\} \\
& =\{M \in \max A: M \notin J+I, M \supseteq I\} \\
& =\{M \in \max A: M \notin J, M \supseteq I\} \\
& =\max J \cap \max A / I .
\end{aligned}
$$

We have a natural isomorphism

$$
J+I / I \rightarrow J / I \cap J
$$

defined by $a+I \rightarrow a+I \cap J$ for $a \in J$. Noting that elements of $J$ vanish outside of $\max J$,

$$
\begin{aligned}
\|a+I\| & =\sup \{|a(M)|: M \in \max A / I\} \\
& =\sup \{|a(M)|: M \in \max J \cap \max A \mid I\} \\
& =\sup \{|a(M)|: M \in \max J-\max I\} \\
& =\sup \{|a(M)|: M \in \max J / I \cap J\}=\|a+I \cap J\|,
\end{aligned}
$$

hence (1.4) is an isometry. The same identifications show that $a+I \geqq 0$ if and only if $a+I \cap J \geqq 0$.

2. $C$ - and $M$-ideals. A simplex space is a $C$-space if it is isomorphic as an ordered Banach space to $C_{0}(X)$, the real continuous functions vanishing at $\infty$ on a locally compact Hausdorff space $X$. For simplex spaces with identity, the following result is essentially due to $H$. Bauer [16].

Proposition 2.1. If $A$ is a simplex space, the following conditions are equivalent:

(1) $A$ is a $C$-space.

(2) The elements of $A$ are continuous on $\max A$, i.e., $M_{A}$ is a homeomorphism.

(3) $E P_{1}(A)$ is closed in $P_{1}(A)$.

(4) $\max A$ is locally compact Hausdorff, and $A=C_{0}(\max A)$.

Proof. We have proved the equivalence of (2)-(4) in [8, Theorem 3.10]. (4) $\Rightarrow$ (1) is trivial. To prove (1) $\Rightarrow(3)$, suppose that $A=C_{0}(X), X$ locally compact Hausdorff. Let $X_{\infty}=X \cup\{\infty\}$ be the one-point compactification of $X . A$ may be identified with the functions in $C\left(X_{\infty}\right)$ vanishing at $\infty$. We define a continuous map $\delta$ of 
$X_{\infty}$ into $P_{1}(A)$ by $\delta(x)(a)=a(x)$. Letting $\partial A$ be the $x \in X_{\infty}$ for which $\|\delta(x)\|=0$ or 1, we have from [8, Lemma 4.1] that $\delta(\partial A)=E P_{1}(A)$. If $x \in X$, we may find $a \in A$ with $\|a\| \leqq 1$ and $a(x)=1$. Thus $\partial A=X_{\infty}$, and $E P_{1}(A)$ is a continuous image of a compact set.

A $C$-ideal in a simplex space $A$ is a closed ideal which is itself a $C$-space or $\{0\}$. A point $x$ in a topological space $X$ is separated if for any $y \neq x$ there are open disjoint neighborhoods $G$ and $H$ of $x$ and $y$, respectively. Equivalently (see [10, p. 67]), if a net $x_{v}$ in $X$ converges to $x$ then it cannot converge to any other point. A subset $S$ of $X$ is separated in $X$ if all of its points are separated in $X$. It is readily verified that if $S$ is such a set, compact subsets of $S$ are closed in $X$. Identifying max $J$ with the open set $\max A-h(J)$,

THEOREM 2.2. Let $J \neq\{0\}$ be a closed ideal in a simplex space $A$. If $J$ is a $C$-ideal, then

(1) Compact subsets of $\max J$ are closed in $\max A$.

(2) $\max J$ is separated in $\max A$.

(3) Elements of $J$ are continuous on $\max A$.

(4) Elements of $A$ restrict to continuous functions on $\max J$.

(5) $Z_{A} \subseteq E P_{1}(A) \cup J^{\perp}$.

Conversely if $J$ satisfies any one of conditions (3)-(5), it is a C-ideal.

To prove this, we need

LeMmA 2.3. Let $A$ be a simplex space, and suppose that a net $p_{v} \in E P_{1}(A)$ converges to a point $q \in P_{1}(A)$. If $Q$ is the smallest closed face containing $q$ and 0 in $P_{1}(A)$, then $M_{p}$ converges to $M_{p}$ for all $p \in E(Q)-\{0\}$.

Proof. If $p \in E(Q)-\{0\}$, and $M_{p_{v}}$ does not converge to $M_{p}$, then there is a closed set $F \subseteq \max A$ such that $M_{p_{v}} \in F$ frequently, but $M_{p} \notin F$. If we let $R$ be the closed face in $P_{1}(A)$ containing 0 with $F=M_{A}(E(R)-\{0\})$, then $p_{v} \in R$ frequently; hence $q \in R$ and $Q \subseteq R$. This contradicts the fact that $p \notin E(R)=R \cap E P_{1}(A)$.

Proof of Theorem 2.2. Referring to diagram (1.2) we are given that $M_{J}$ is a homeomorphism. If $K \subseteq \max J$ is compact, $M_{J}^{-1}(K)$ is compact, and from Lemma 1.1, $K_{1}=\rho_{E}^{-1} M_{J}^{-1}(K)$ is compact in $E P_{1}(A)-J^{\perp}$. It follows from [8, Corollary 3.5] that $\eta_{A}^{-1}(K)=M_{A}\left(K_{1}\right)$ is closed in $\max A-h(J)$.

In the remainder of the proof, we identify $\max J$ and $\max A-h(J)$. To prove (2), suppose that $M_{1} \in \max J, M_{2} \in \max A$, and $M_{1} \neq M_{2}$. If $M_{2} \in \max J$, we may separate $M_{1}$ and $M_{2}$ since $\max J$ is locally compact Hausdorff ((4) of Proposition 2.1). If $M_{2} \notin \max J$, let $G$ be an open neighborhood of $M_{1}$ with compact relative closure in $\max J$. From (1), the relative closure must be closed in $\max A$. Thus $G$ and the complement of its closure separate $M_{1}$ and $M_{2}$.

To prove (3), suppose that $u \in J$ and $M_{v} \rightarrow M$ is a convergent net in max $A$. If $M \in \max J$, then eventually $M_{v} \in \max J$, and $u\left(M_{v}\right) \rightarrow u(M)$ follows from (4) of Proposition 2.1. If $M \notin \max J$, then $u(M)=0$. Given $\varepsilon>0$, the set

$$
K=\{N \in \max A:|u(N)| \geqq \varepsilon\}
$$


is a compact subset of $\max J$ [7, Proposition 4.5]; hence, from (1), it is closed in $\max A$. Since $M \notin K$, we must eventually have $M_{v} \notin K$, hence $\left|u\left(M_{v}\right)\right|<\varepsilon$. We conclude that $u\left(M_{v}\right) \rightarrow u(M)$.

Turning to (4), it suffices to prove that if $a \in A$, and $G$ is an open subset with compact relative closure in $\max J$, then $a$ is continuous on $G$. Let $u \in J=C_{0}(\max J)$ be such that $u(M)=1$ for $M \in G$. $u$ exists since $\max J$ is locally compact. From (3), $u$ is continuous on $\max A$; hence $u a \in A$ [8, Theorem 2.1]. Since $u a(M)=0$ for $M \notin \max J, u a \in J$, and $u a$ is also continuous on $\max A$. Since $u a(M)=a(M)$ for $M \in G, a$ is continuous on $G$.

To prove (5), suppose that $p_{v}$ is a net in $E P_{1}(A)$ converging to $q \in P_{1}(A)$, and that $q \notin J^{\perp}$. Let $Q$ be the smallest closed face in $P_{1}(A)$ containing $q$ and 0 . Since $Q \nsubseteq J^{\perp}$, we may choose

$$
p_{0} \in E(Q)-J^{\perp} \subseteq E P_{1}(A)-J^{\perp} .
$$

From Lemma 2.3, if $p \in E(Q)-\{0\}$, then $M_{p_{v}} \rightarrow M_{p}$, and in particular $M_{p_{v}}$ $\rightarrow M_{p_{0}} \in \max J$. Since $M_{p_{0}}$ is separated in $\max A, E(Q)-\{0\}=\left\{p_{0}\right\}$ and $Q$ is the line segment joining 0 and $p_{0}$. Let $q=\alpha p_{0}, 0<\alpha \leqq 1$, and choose $u \in J$ with $u\left(M_{p_{0}}\right) \neq 0$. Then

$$
u\left(M_{p_{v}}\right)=p_{v}(u) \rightarrow q(u)=\alpha p_{0}(u),
$$

and since $u$ is continuous on $\max J$,

$$
u\left(M_{p_{v}}\right) \rightarrow u\left(M_{p_{0}}\right)=p_{0}(u) .
$$

Thus $\alpha=1$, and $q=p_{0} \in E P_{1}(A)-J^{\perp}$.

That (3) and (4) imply $J$ is a $C$-ideal is apparent from (2) of Proposition 2.1. Since $\rho_{1}\left(E P_{1}(A)\right)=E P_{1}(J)$ and $Z_{A}=\left[E P_{1}(A)\right]^{-}$is compact, we have from (5),

$$
\left[E P_{1}(J)\right]^{-}=\rho_{1}\left(Z_{A}\right) \subseteq \rho_{1}\left(E P_{1}(A)\right) \cup \rho_{1}\left(J^{\perp}\right)=E P_{1}(J),
$$

and (3) of Proposition 2.1 implies that $J$ is a $C$-ideal.

At this point it would seem reasonable to define an " $M$-ideal" in a simplex space to be a closed ideal which is itself an $M$-space or $\{0\}$. However, since the analogue of Theorem 2.2 is not true for such ideals (see \$7.1), it is necessary to consider a "relative" property. We say that a closed ideal $J$ in a simplex space $A$ is an $M$-ideal if $J=\{0\}$, or $J$ satisfies one of the conditions of Theorem 2.5 (see below).

Let $F(\max A)$ be the uniformly bounded functions on $\max A$ with the uniform norm. $F(\max A)$ is an algebra and a lattice ordered Banach space (in fact, a $C$ space) under the usual operations. As in [8, §2], we may identify $A$ as a Banach space with a closed subspace $A^{\prime}$ of $F(\max A)$, since

$$
\|a\|=\sup \{|a(M)|: M \in \max A\}
$$

(this is a simple consequence of the Krein-Milman Theorem-see the proof of 
[7, Proposition 4.5]). The ordering on $A$ coincides with the relative ordering on $A^{\prime}$ since

$$
\begin{aligned}
A^{+} & =\left\{a \in A: p(a) \geqq 0 \text { for all } p \in P_{1}(A)\right\} \\
& =\left\{a \in A: p(a) \geqq 0 \text { for all } p \in E P_{1}(A)-\{0\}\right\} \\
& =\{a \in A: a(M) \geqq 0 \text { for all } M \in \max A\} .
\end{aligned}
$$

The first equality follows from [7, Theorem 2.2]. The second is due to the fact that if $\alpha$ is the least value $a$ assumes on $P_{1}(A)$,

$$
Q=\left\{p \in P_{1}(A): p(a)=\alpha\right\}
$$

is a closed nonempty face in $P_{1}(A)$; hence if $p(a)<0$ for some $p \in P_{1}(A)$, the same is true for some $p^{\prime} \in E P_{1}(A)$.

When we use the symbols $\wedge$ and $\vee$ below, we are taking minima and maxima in the lattice $F(\max A)$. We let $M(A)$ be the elements $a \in A^{+}$such that for all $b \in A^{+}, a \wedge b \in A$. We recall that $R P_{1}(A)$ consists of the extremal rays of $P_{1}(A)$ containing 0 , i.e., the line segments joining 0 to extreme points.

The following is proved in [2, pp. 99-100].

LEMMA 2.4. Let $K$ be a compact convex set in a locally convex space. If $Q$ is a closed face in $K$ and $\mu$ is a probability measure with resultant $r(\mu)$ in $Q$, then supp $\mu \subseteq Q$.

TheOREM 2.5. Suppose that $J \neq\{0\}$ is a closed ideal in a simplex space A. Then the following are equivalent:

(1) $J^{+} \subseteq M(A)$.

(2) $\max J$ is separated in $\max A$.

(3) $Z_{A} \subseteq R P_{1}(A) \cup J^{\perp}$.

Proof. (1) $\Rightarrow$ (2). Suppose that $M_{1} \in \max J$ (the latter regarded as a subset of $\max A$ ) and that $M_{2} \in \max A, M_{1} \neq M_{2}$. Choose $u \in J^{+}$with $u\left(M_{1}\right) \neq 0$ and $u\left(M_{2}\right)$ $=0$. This exists since if $M_{2} \in \max J, M_{1} \cap J$ and $M_{2} \cap J$ are distinct maximal ideals in $J$; hence we may select $u \in J \cap M_{2}-J \cap M_{1}$. We may assume $u \geqq 0$ since $J \cap M_{2}$ is a closed ideal, and thus is positively generated. If $M_{2} \notin \max J$, we may take any $u \in J^{+}-M_{1}$. Similarly, let $a \in A^{+}$be such that $a\left(M_{1}\right)=0, a\left(M_{2}\right) \neq 0$. Since $u \wedge a \in A$, the sets

$$
\begin{aligned}
& \{M: u(M)>a(M)\}=\{M:(u-u \wedge a)(M)>0\}, \\
& \{M: a(M)>u(M)\}=\{M:(a-u \wedge a)(M)>0\},
\end{aligned}
$$

are open [7, Proposition 4.7], and separate $M_{1}$ and $M_{2}$.

(2) $\Rightarrow(3)$. Suppose that $p_{v}$ is a net in $E P_{1}(A)$ converging to $q \in P_{1}(A)$, and that $q \notin J^{\perp}$. Letting $Q$ be the smallest closed face in $P_{1}(A)$ containing $q$ and 0 , we have $Q$ is the line segment joining 0 to an element $p_{0} \in E P_{1}(A)-\{0\}$ (see the proof of (5) in Theorem 2.2). Thus $q \in R P_{1}(A)$. 
(3) $\Rightarrow$ (1). From [8, Corollary 2.5], we may identify $A$ with $\mathscr{A}_{0}\left(Z_{A}\right)$, the affine continuous functions on $Z_{A}$, vanishing at 0 . If $u \in J^{+}$, and $a \in A^{+}$, consider the function $f(p)=\min \{u(p), a(p)\}$ defined on $Z_{A} . f$ is continuous and vanishes at 0 . To show it is affine, say that $p \in Z_{A}$ and $\mu_{p}$ is the extremal measure with resultant $p$. If $p \in J^{\perp}$, then from Lemma 2.4 the support of $\mu_{p}$ is contained in the closed face $J^{\perp}$. Since $u$ is zero on $J^{\perp}$, and $a \geqq 0$,

$$
\mu_{p}(f)=0=f(p) .
$$

If $p \notin J^{\perp}$, we have $p=\alpha q$, where $q \in E P_{1}(A)-\{0\}, 0<\alpha \leqq 1$. Then $\mu_{p}=\alpha \delta_{q}+(1-\alpha) \delta_{0}$, and since $u, a \in \mathscr{A}_{0}\left(Z_{A}\right)$,

$$
\begin{aligned}
\mu_{p}(f) & =\alpha f(q)=\alpha \min \{u(q), a(q)\} \\
& =\min \{\alpha u(q), \alpha a(q)\}=\min \left\{\mu_{p}(u), \mu_{p}(a)\right\} \\
& =\min \{u(p), a(p)\}=f(p) .
\end{aligned}
$$

It follo vs that $f \in A$, and if $M \in \max A$,

$$
f(M)=f\left(p_{M}\right)=\min \left\{u\left(p_{M}\right), a\left(p_{M}\right)\right\}=u \wedge a(M) .
$$

If $u \in M(A)$, one cannot conclude that $u \wedge a \in A$ for nonpositive $a \in A$. P. Taylor has shown that if such an element $u$ exists, then $A$ is an $M$-space.

It is clear from (3) of Theorem 2.5 and Rogalski's characterization of $M$-spaces (see [15, Theorem 2]) that a simplex space is an $M$-ideal in itself if and only if it is an $M$-space. Thus we may delete the condition of separability in [8, Theorem 3.8] (this result has been independently obtained by F. Perdrizet).

COROLlary 2.6. If $A$ is a simplex space the following conditions are equivalent:

(1) $A$ is an $M$-space.

(2) $\max A$ is Hausdorff.

(3) $Z_{A} \subseteq R P_{1}(A)$.

The following technical results will play an important role in Theorem 5.2. If $A$ is a simplex space, define $\bar{M}_{A}: R P_{1}(A)-\{0\} \rightarrow \max A$ by

$$
\bar{M}_{A}(p)=M_{A}(p /\|p\|) \text {. }
$$

Let $I \neq A$ and $J \neq\{0\}$ be closed ideals in $A$. Then the following diagrams commute:

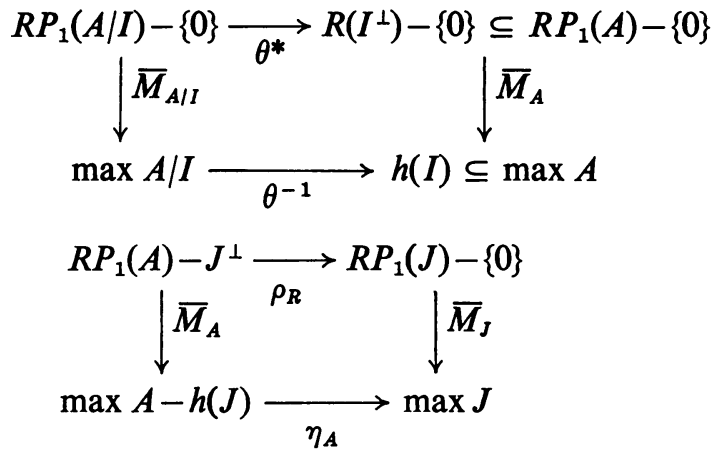


where $\rho_{R}$ is the restriction of $\rho_{1}: P_{1}(A) \rightarrow P_{1}(J)$. Since $\rho_{1}$ restricts to a one-to-one map on $E P_{1}(A)-J^{\perp}$, the same is true on $R P_{1}(A)-J^{\perp}$. Again it is not clear that $\rho_{R}$ is a homeomorphism, but we have the following analogue of Lemma 1.1.

Lemma 2.7. Suppose that $J$ is an $M$-ideal in a simplex space $A$. If $K$ is a compact subset of $R P_{1}(J)-\{0\}$, then $\rho_{R}^{-1}(K)$ is compact in $R P_{1}(A)-J^{\perp}$.

Proof. Since $J$ is an $M$-ideal, upon taking closures in $P_{1}(A)$ we have

$$
\left[E P_{1}(A)\right]^{-} \subseteq R P_{1}(A) \cup J^{\perp}
$$

$[0,1]\left[E P_{1}(A)\right]^{-}$is compact, hence closed in $P_{1}(A)$, and

$$
\left[R P_{1}(A)\right]^{-} \subseteq[0,1]\left[E P_{1}(A)\right]^{-} \subseteq R P_{1}(A) \cup J^{\perp}
$$

It follows that

$$
\left[\rho_{R}^{-1}(K)\right]^{-} \subseteq R P_{1}(A) \cup J^{\perp}
$$

But $\rho_{1}$ is continuous on $P_{1}(A)$ and $K$ is closed in $P_{1}(J)$; hence

$$
\rho_{1}\left(\left[\rho_{R}^{-1}(K)\right]^{-}\right) \subseteq K
$$

Since $0 \notin K$, we conclude from (2.3) that

$$
\left[\rho_{R}^{-1}(K)\right]^{-} \subseteq R P_{1}(A)-J^{\perp} .
$$

$\rho_{R}=\rho_{1} \mid R P_{1}(A)-J^{\perp}$ is a bijection, and we have from (2.4) that $\rho_{R}^{-1}(K)=\left[\rho_{R}^{-1}(K)\right]^{-}$; hence $\rho_{R}^{-1}(K)$ is compact.

3. Composition series of open sets. We must consider various properties that open subsets $G$ of a topological space $X$ can satisfy, such as

$M: G$ is separated in $X$.

$M L C: G$ is separated in $X$ and locally compact in the relative topology.

If $X=\max A$ with $A$ a simplex space, we may reformulate $M$ (see (2) of Theorem 2.5).

$M: G=\max J$ where $J$ is an $M$-ideal in $A$.

We also have the property

$C: G=\max J$ where $J$ is a $C$-ideal in $A$.

In order to make our discussion precise, we define a property or topological property $P$ to be a family of topological spaces $\mathscr{P}$, together with a collection $P_{X}$ of open subsets for each $X \in \mathscr{P}$. We will assume that if $X \in \mathscr{P}$, then with their relative topologies, the nonempty open subsets and the nonempty closed subsets of $X$ also lie in $\mathscr{P}$. If $G \in P_{X}$, we write $G \subseteq_{P} X$ and say that $G$ is a $P$-set in $X$. If $X \subseteq_{P} X$, we say that $X$ is a $P$-space. For $M$ and $M L C$ we let $\mathscr{P}$ be all topological spaces, and for $C, \mathscr{P}$ is the locally closed subsets of a given structure space (see $\S 4$ ).

If $P$ is a property, we say that it inductive if for $X \in \mathscr{P}$ and $F \neq \varnothing$ closed in $X$, $I_{1} . G \subseteq_{P} X$ implies that $G \cap F \subseteq_{P} F$.

It is clear that $M$ and $M L C$ are inductive, and we shall prove the same for $C$ in $\S 4$. 
Given a property $P$ and a space $X \in \mathscr{P}$, we say that an open subset $G$ of $X$ is $G P$ in $X$, written $G \subseteq_{G P} X$, if for each closed subset $F$ of $X$, either $G \cap F=\varnothing$ or $G \cap F$ contains a nonempty relatively open $P$-subset of $F$. $G P$ is again a property, and we will use the terminology "generalized $P$ " or "almost $P$ ". From above, $X$ is a $G P$-space if $X \subseteq_{G P} X$. We say $X$ is an NP-space (or "nowhere $P$ ") if it contains no nonempty open $P$-set.

Proposition 3.1. Let $P$ be an inductive property. If $X \in \mathscr{P}$, there is a largest ${ }^{\circ} G P$ open subset $G$ in $X$, and $X-G$ is NP or empty. Furthermore there is a collection of distinct open subsets $G_{\gamma}$ indexed by ordinals $0 \leqq \gamma \leqq \gamma_{0}$, such that

(1) $G_{0}=\varnothing, G_{\gamma_{0}}=G$.

(2) If $\gamma<\gamma_{0}$, then $G_{\gamma}$ is a proper subset of $G_{\gamma+1}$ and $G_{\gamma+1}-G_{\gamma} \subseteq{ }_{P} X-G_{\gamma}$.

(3) If $\gamma \leqq \gamma_{0}$ is a limit ordinal, then $G_{\gamma}=\bigcup_{\beta<\gamma} G_{\beta}$.

Proof. Given $X$, let $G_{0}=\varnothing$. Suppose that we have defined open $G_{\beta}$ for ordinals $\beta<\gamma$. If $\gamma$ is a limit ordinal, let $G_{\gamma}=\bigcup_{\beta<\gamma} G_{\beta}$. If $\gamma$ is not a limit ordinal, let $G_{\gamma}=G_{\gamma-1}$ if $G_{\gamma-1}=X$ or $X-G_{\gamma-1}$ is $N P$. Otherwise let $G_{\gamma}^{\prime} \neq \varnothing$ be a relatively open $P$-set in $X-G_{\gamma-1}$, and let $G_{\gamma}=G_{\gamma}^{\prime} \cup G_{\gamma-1}$. Let $\gamma_{0}$ be the first ordinal with $G_{\gamma_{0}}=G_{\gamma_{0}+1}$, ard let $G=G_{\gamma_{0}}$. Then $X-G$ is $N P$ or $\varnothing$. It is clear that the $G_{\gamma}$ satisfy (2) and (3).

Since $G_{0}=\varnothing, G_{0} \subseteq_{G P} X$. Suppose that we have proved $G_{\beta} \subseteq_{G P} X$ for all $\beta<\gamma$, and that $F$ is closed in $X$ with $F \cap G_{\gamma} \neq \varnothing$. Letting $\beta_{0} \leqq \gamma$ be the first ordinal with $F \cap G_{\beta_{0}}$ $\neq \varnothing$, it is clear from (3) that $\beta_{0}$ is not a limit ordinal. From (2) and $I_{1}, F \cap$ $\left(G_{\beta_{0}}-G_{\beta_{0}-1}\right)=F \cap G_{\beta_{0}}$ is a $P$-set in $F \cap\left(X-G_{\beta_{0}-1}\right)=F$. Thus $G_{\gamma} \subseteq{ }_{G P} X$.

Suppose that $H$ is a $G P$ open set in $X$. If $H \nsubseteq G$, then $H \cap(X-G)$ must contain a nonempty, relatively open $P$-subset of $X-G$. This contradicts the fact that $X-G$ is $N P$.

We will refer to a sequence $G_{y}$ having the above properties as a $P$-composition series.

If $P$ is inductive, then letting $X=G$ in $I_{1}$, we have that nonempty closed subsets of a $P$-space are $P$-spaces. Since for any property $P, G P$ is inductive (see below), closed subsets of $G P$-spaces are again $G P$-spaces. To obtain an analogous argument for open sets, we say that a property $P$ is strongly inductive if given $X \in \mathscr{P}$, $G_{1}, G_{2}$ open and $F$ closed in $X$, it satisfies $I_{1}$ and

$I_{2} . G_{1} \subseteq G_{2} \subseteq X$ and $G_{1} \subseteq{ }_{P} X$ imply $G_{1} \subseteq{ }_{P} G_{2}$.

$I_{3} . G_{1} \subseteq G_{2} \subseteq{ }_{P} X$ implies $G_{1} \subseteq_{P} X$.

$I_{4} . G_{1} \subseteq{ }_{P} F \subseteq X$ implies $G_{1} \subseteq_{P} X$.

It is readily verified that $M$ and $M L C$ are strongly inductive. We shall prove the same for $C$ in $\$ 4$. Note that letting $G_{1}=G_{2}$ in $I_{2}$, a nonempty open $P$-set must itself be a $P$-space. Letting $G_{2}=X$ in $I_{3}$, it follows that a nonempty open subset of a $P$-space is a $P$-space.

LEMMA 3.2. If $P$ is any property, then $G P$ is inductive. If $P$ is strongly inductive, then so is GP. 
Proof. Let $P$ be an arbitrary property, and suppose that $G \subseteq_{G P} X$ and $F$ is closed in $X$. A closed subset of $G \cap F$ must have the form $G \cap F^{\prime}, F^{\prime}$ closed in $X, F^{\prime} \subseteq F$. Since $G \subseteq_{G P} X$, if $G \cap F^{\prime} \neq \varnothing$, then $G \cap F^{\prime}$ contains a nonempty relatively open $P$-set in $F^{\prime}$. Thus $G \cap F \subseteq{ }_{G P} F$.

Suppose that $P$ is strongly inductive. If $G_{1} \subseteq G_{2} \subseteq X$ and $G_{1} \subseteq_{G P} X$, then given $F$ closed in $G_{2}, F=F^{\prime} \cap G_{2}, F^{\prime}$ closed in $X$, and $F \cap G_{1}=F^{\prime} \cap G_{1}$. Choose $E$ relatively open in $F^{\prime} \cap G_{1}$ with $\varnothing \neq E \subseteq{ }_{P} F^{\prime}$. We have $E \subseteq F \subseteq F^{\prime}$, and $F$ is relatively open in $F^{\prime}$. From $I_{2}$ for $P, E \subseteq \subseteq_{P} F$, and we conclude $G P$ satisfies $I_{2}$.

If $G_{1} \subseteq G_{2} \subseteq G P$, then since $G P$ is inductive, $G_{1} \cap F \subseteq G_{2} \cap F \subseteq_{G P} F$. Thus to prove $G_{1} \subseteq_{G P} X$, all we need show is that if $G_{1} \neq \varnothing$, then $G_{1}$ contains a nonempty $P$-subset of $X$. Letting $X_{\gamma}, 0 \leqq \gamma \leqq \gamma_{0}$ be a $P$-composition series for $X$, we have from Proposition 3.1 that $G_{2} \subseteq X_{\gamma_{0}}$. Let $\gamma_{1} \leqq \gamma_{0}$ be the first ordinal with $G_{1} \cap X_{\gamma_{1}} \neq \varnothing$. Then $\gamma_{1}$ is not a limit ordinal. From (2) of Proposition 3.1 and $I_{3}$ for $P$,

$$
G_{1} \cap X_{\gamma_{1}}=G_{1} \cap\left(X_{\gamma_{1}}-X_{\gamma_{1}-1}\right) \subseteq_{P} X-X_{\gamma_{1}-1} .
$$

Thus from $I_{4}$ for $P, G_{1} \cap X_{\gamma_{1}} \subseteq_{P} X$, and $G P$ satisfies $I_{3}$.

Suppose $G_{1} \subseteq G P F \subseteq X, G_{1}$ open and $F$ closed in $X$. If $F^{\prime}$ is closed in $X, G_{1} \cap F^{\prime}$ $\subseteq_{G P} F \cap F^{\prime} \subseteq F^{\prime}$. Thus to show $G_{1} \subseteq_{G P} X$, it suffices to show that if $G_{1} \neq \varnothing$, it contains a nonempty $P$-set in $X$. This follows from $I_{4}$ for $P$.

CoROllaRY 3.3. If $P$ is strongly inductive, then open and closed subsets of a GP-space are GP-spaces.

A topological space is said to be $K_{\sigma}$ if it is a countable union of compact sets, and locally $K_{\sigma}$ if the $K_{\sigma}$-open subsets form a basis. It is clear that both open and closed subsets of a locally $K_{\sigma}$ space are locally $K_{\sigma}$.

Lemma 3.4. If $A$ is a simplex space, then $\max A$ is locally $K_{\sigma}$.

Proof. Let $M_{0}$ be a point in $\max A$, and $G$ be an open neighborhood of $M_{0}$. If $J$ is the closed ideal corresponding to $G$, we may choose $u \in J^{+}$with $u\left(M_{0}\right) \neq 0$. From [7, Proposition 4.5],

$$
G^{\prime}=\{M: u(M) \neq 0\}=\bigcup_{n}\{M: u(M) \geqq 1 / n\}
$$

is a $K_{\sigma}$ open set with $M_{0} \in G^{\prime} \subseteq G$.

Applying this to $C$-spaces, we have the well-known result that locally compact Hausdorff spaces are locally $K_{\sigma}$. We note that a similar proof shows that the primitive ideal space of a $C^{*}$-algebra is locally $K_{\sigma}$.

We recall that a topological space $X$ is second category if countable intersections of dense open sets are nonempty. $X$ is Baire if such intersections are dense, or equivalently, each open set is second category. We say that $X$ is strongly Baire if each closed set is Baire, or equivalently, each locally closed set (i.e., the intersection of an open and a closed set) is second category in itself. In contrast to 
$C^{*}$-algebras, the structure space of a simplex space need not be strongly Baire $[7, \S 5]$, or locally compact [8, Theorem 4.3$]$.

Lemma 3.5. Suppose that $X$ is locally $K_{\sigma}$. Then the following are equivalent:

(1) $X$ is strongly Baire and almost separated (i.e., GM).

(2) $X$ is almost locally compact separated (i.e., GMLC).

Proof. (1) $\Rightarrow$ (2). If $F \neq \varnothing$ is a closed subset of $X$, let $G \neq \varnothing$ be a relatively open separated subset. Since $F$ is locally $K_{\sigma}$, we may choose a $K_{\sigma}$ open set $G^{\prime} \neq \varnothing$ with $G^{\prime} \subseteq G$. Let $G^{\prime}=\bigcup C_{n}$, with $C_{n}$ compact. Since $G^{\prime}$ is second category, there is an $n_{0}$ with $H=$ int $C_{n_{0}} \neq \varnothing$. Since $G$ is separated in $F, C_{n_{0}}$ is closed in $F$, hence from $I_{4}, H \subseteq_{M L C} C_{n_{0}} \subseteq F$ implies $H \subseteq_{M L C} F$.

(2) $\Rightarrow(1)$. Since $M L C$ is strongly inductive, we have from Corollary 3.3 that open and closed subsets of an almost locally compact separated space have the same property. Thus it suffices to show that if $X$ is such a space, then $X$ is second category. Let $G \neq \varnothing$ be a locally compact Hausdorff subset of $X$. If $G_{n}, n=1,2, \ldots$ are dense open sets in $X$, then $G \cap G_{n}$ are dense open sets in $G$. Since any locally compact Hausdorff space is second category, it follows that $\bigcap_{n} G \cap G_{n} \neq \varnothing$, hence $\bigcap_{n} G_{n} \neq \varnothing$.

4. Composition series of closed ideals. By analogy to $\S 3$, we must consider various properties that closed ideals $J$ in a simplex space $A$ can satisfy, such as

$M: J$ is an $M$-ideal in $A$.

$M L C: J$ is an $M$-ideal in $A$ and $\max J$ is locally compact.

$C: J$ is a $C$-ideal in $A$.

A simplex property is an association with each simplex space $A$ of a family of closed ideals $P_{A}$. If $J \in P_{A}$ we write $J \subseteq{ }_{P} A$ and say that $J$ is a $P$-ideal in $A$. If $A \subseteq_{P} A$ we say that $A$ is a $P$-space. We will consider only simplex properties that are preserved under isomorphisms, i.e., if $\theta: A \rightarrow A^{\prime}$ is an isomorphism onto, then $J \subseteq_{P} A$ if and only if $\theta(J) \subseteq_{P} \theta(A)$.

We note that $M$ and $M L C$ are derived from corresponding topological properties. If $P$ is any such property, and $\mathscr{P}$ contains the structure spaces of simplexes, we obtain a simplex property by letting $J \subseteq_{P} A$ if $\max J \subseteq_{P} \max A$.

Conversely, a simplex property defines a collection of topological properties. For each simplex space $A_{0}$, let $\mathscr{P}_{A_{0}}$ be all the nonempty locally closed subsets of $\max A_{0}$. Each $X$ in $\mathscr{P}_{A_{0}}$ has the form $G_{0} \cap F_{0}$ with $G_{0}$ open, $F_{0}$ closed in $\max A_{0}$. We let

$$
A\left(G_{0}, F_{0}\right)=\theta_{I_{0}}\left(J_{0}\right)=J_{0}+I_{0} / I_{0},
$$

where $I_{0} \neq A_{0}, J_{0} \neq\{0\}$ are the closed ideals in $A_{0}$ with $F_{0}=\max A_{0} / I_{0}, G_{0}=\max J_{0}$. From (1.3) we have

$$
X=G_{0} \cap F_{0}=\max A\left(G_{0}, F_{0}\right)
$$


when the latter is considered a subset of $\max A_{0}$. The relatively open subsets $G$ of $X$ have the form max $J$, where $J$ is a closed ideal in $A\left(G_{0}, F_{0}\right)$. We say $G$ is a $P$-set in $X$, written $G \subseteq_{P} X$, if $J \subseteq_{P} A\left(G_{0}, F_{0}\right)$. This definition does not depend upon $\left(G_{0}, F_{0}\right)$ since if $G_{0} \cap F_{0}=G_{1} \cap F_{1}$, then there is an isomorphism of $A\left(G_{0}, F_{0}\right)$ onto $A\left(G_{1}, F_{1}\right)$ which identifies the ideals corresponding to a given relatively open subset of $X$.

We say that a simplex property $P$ is inductive if for each simplex space $A$ and closed ideals $I \neq A, J$

$I_{1}^{\prime}: J \subseteq_{P} A$ implies $J+I / I \subseteq_{P} A / I$.

We say $P$ is strongly inductive if in addition,

$I_{2}^{\prime}: J_{1} \subseteq J_{2} \subseteq A$ and $J_{1} \subseteq_{P} A$ imply $J_{1} \subseteq_{P} J_{2}$.

$I_{3}^{\prime}: J_{1} \subseteq J_{2} \subseteq_{P} A$ implies $J_{1} \subseteq_{P} A$.

$I_{4}^{\prime}: J_{1} \cap I=\{0\}$ and $J_{1}+I / I \subseteq_{P} A / I$ imply $J_{1} \subseteq_{P} A$.

We have:

LEMMA 4.1. A simplex property is (strongly) inductive if and only if the corresponding topological properties are (strongly) inductive.

Proof. Let $A_{0}$ be a simplex space, and $G \subseteq G_{0}$ and $F \subseteq F_{0}$ be open and closed subsets of $\max A_{0}$, respectively. The open and closed subsets of $X=G_{0} \cap F_{0}$ have the form $G \cap F_{0}$ and $G_{0} \cap F$, respectively. On the other hand, the closed ideals in $A=A\left(G_{0}, F_{0}\right)$ have the form $J=A\left(G, F_{0}\right)$. We may restate $I_{1}$ of $\S 3$ for $X=G_{0} \cap F_{0}$ as follows:

$$
G \cap F_{0} \subseteq_{P} G_{0} \cap F_{0} \text { implies } G \cap F \subseteq_{P} G_{0} \cap F,
$$

or, from the definition of $\subseteq_{P}$ for locally closed sets,

$$
A\left(G, F_{0}\right) \subseteq_{P} A\left(G_{0}, F_{0}\right) \text { implies } A(G, F) \subseteq_{P} A\left(G_{0}, F\right) .
$$

But letting $I=A\left(G_{0}-F, F_{0}\right)$, there is a natural isomorphism of $A\left(G_{0}, F\right)$ onto $A / I=A\left(G_{0}, F_{0}\right) / I$, which carries $A(G, F)$ onto $J+I / I=A\left(G, F_{0}\right)+I / I$. Thus

$$
J \subseteq_{P} A \text { implies } J+I / I \subseteq_{P} A / I,
$$

and noting that $I$ is a general closed ideal in $A$, we have the equivalence of $I_{1}$ and $I_{1}^{\prime}$.

The equivalences of $I_{2}, I_{2}^{\prime}$ and $I_{3}, I_{3}^{\prime}$ are trivial. Letting $G_{0}, F_{0}, X, G, F$ be as above, we may restate $I_{4}$ :

$$
G \cap F_{0} \subseteq_{P} G_{0} \cap F \subseteq G_{0} \cap F_{0} \text { implies } G \cap F_{0} \subseteq_{P} G_{0} \cap F_{0}
$$

We have $G \cap F_{0} \subseteq G_{0} \cap F$ if and only if

$$
\left[G \cap F_{0}\right] \cap\left[\left(G_{0}-F\right) \cap F_{0}\right]=\varnothing,
$$

or regarding $A\left(G, F_{0}\right)$ and $I=A\left(G_{0}-F, F_{0}\right)$ as ideals in $A\left(G_{0}, F_{0}\right)$,

$$
A\left(G, F_{0}\right) \cap I=\{0\} \text {. }
$$


Assuming $G \cap F_{0} \subseteq G_{0} \cap F$, we have $G \cap F_{0} \subseteq{ }_{P} G_{0} \cap F$ if and only if $G \cap F$ $\subseteq_{P} G_{0} \cap F$, i.e., $A(G, F) \subseteq_{P} A\left(G_{0}, F\right)$. Using the natural isomorphism of the previous paragraph, we have $G \cap F_{0} \subseteq_{p} G_{0} \cap F$ if and only if we have (4.1) and $A\left(G, F_{0}\right)+I / I \subseteq_{P} A\left(G_{0}, F_{0}\right) / I$. Letting $A=A\left(G_{0}, F_{0}\right)$ and $J=A\left(G, F_{0}\right)$, we have the equivalence of $I_{4}$ and $I_{4}^{\prime}$.

Given a simplex property $P$, we say that a closed ideal $J$ is GP in a simplex space $A$, written $J \subseteq_{G P} A$, if for all closed ideals $I \neq A, J \subseteq I$ or $J+I / I$ contains a nonzero closed $P$-ideal in $A / I$. We say that $A$ is an $N P$-space if it contains no nonzero $P$-ideal. In terms of the corresponding topological property, $J \subseteq_{G P} A$ if and only if $\max J$ $\subseteq_{G P} \max A$, and $A$ is $N P$ if and only if that is the case for $\max A$. We have:

Proposition 4.2. Let $P$ be an inductive simplex property. Then in each simplex space $A$ there is a largest GP-ideal $J$. If $J \neq A$, then $A / J$ is NP. There is a collection of distinct closed ideals $J_{\gamma}$ indexed by ordinals $0 \leqq \gamma \leqq \gamma_{0}$ such that

(1') $J_{0}=\{0\}, J_{\gamma_{0}}=J$.

(2') If $\gamma<\gamma_{0}$, then $J_{\gamma}$ is a proper subset of $J_{\gamma+1}$, and $J_{\gamma+1} / J_{\gamma} \subseteq_{P} A / J_{\gamma}$.

(3') If $\gamma \leqq \gamma_{0}$ is a limit ordinal, then $J_{\gamma}=\left[\bigcup_{\beta<\gamma} J_{\beta}\right]^{-}$.

If $P$ is strongly inductive, then ideals and quotients of GP-spaces are again GPspaces.

Proof. Using the corresponding topological property for locally closed subsets of $X=\max A$, let $G_{\gamma}$ and $G$ be open sets as described in Proposition 3.1, and $J_{\gamma}$, $J$ the closed ideals in $A$ with $G_{\gamma}=\max J_{y}, G=\max J$. Then $\left(1^{\prime}\right)-\left(3^{\prime}\right)$ follow from (1)-(3) and the following remarks. From the inclusion

$$
G_{\gamma+1}-G_{\gamma}=G_{\gamma+1} \cap\left(X-G_{\gamma}\right) \subseteq_{P} X-G_{\gamma},
$$

we conclude that

$$
A\left(G_{\gamma+1}, X-G_{\gamma}\right) \subseteq_{P} A\left(X, X-G_{\gamma}\right),
$$

i.e., $J_{\gamma+1} / J_{\gamma} \subseteq_{P} A / J_{\gamma}$. Let $\gamma$ be a limit ordinal. Since the $G_{\beta}, \beta<\gamma$, form an increasing sequence, the same is true for $J_{\beta}$, and $\bigcup_{\beta<\gamma} J_{\beta}=\sum_{\beta<\gamma} J_{\beta}$. The latter and its closure $I$ are ideals (see $[7, \S 3]$ ), and

$$
\max I=X-h(I)=X-\bigcap_{\beta<\gamma} h\left(J_{\beta}\right)=\bigcup_{\beta<\gamma} \max J_{\beta}=\max J_{\gamma} ;
$$

hence $I=J_{y}$.

The remainder of the proposition follows from Corollary 3.3.

We shall refer to a sequence of closed ideals $J_{y}$ having the above properties as a $P$-composition series for $A$.

We say that a simplex property $P$ is absolute if closed ideals are $P$-ideals if and only if they are themselves $P$-spaces, i.e., if $J$ is a closed ideal in $A$,

$$
J \subseteq_{P} A \text { if and only if } J \subseteq_{P} J .
$$

If this is not the case, we say that $P$ is relative. From its definition $C$ is absolute, 
whereas $M$ and $M L C$ are relative (see $\S 7.1$ ). If $P$ is absolute, $I_{1}^{\prime}$ and $I_{3}^{\prime}$ may be restated

$$
J \subseteq_{P} J \text { implies } J / I \cap J \subseteq_{P} J / I \cap J
$$

and

$$
J_{1} \subseteq J_{2} \text { and } J_{2} \subseteq_{P} J_{2} \text { imply } J_{1} \subseteq_{P} J_{1},
$$

respectively, and both $I_{2}^{\prime}$ and $I_{4}^{\prime}$ are vacuous. Thus an absolute property is (strongly) inductive if and only if (ideals and) quotients of $P$-spaces are $P$-spaces. Also it is clear that if $P$ is absolute, the same is true for $G P$.

Proposition 4.3. The simplex properties $M, M L C$, and $C$ are strongly inductive.

Proof. Since the topological properties $M$ and $M L C$ are strongly inductive, we have from Proposition 4.1 that the same is true for the corresponding simplex properties. From the above discussion, it suffices to show that if $J \neq\{0\}$ and $I \neq A$ are closed ideals in a $C$-space $A$, then $J$ and $A / I$ are $C$-spaces. But $J$ and $A / I$ may be identified with the functions in $A$ vanishing off $\max J$ and the restrictions of functions in $A$ to $\max A / I$. In both cases, elements are continuous on the structure space; hence, from (2) of Proposition 2.1, $J$ and $A / I$ are $C$-spaces.

From Propositions 4.2 and 4.3 we conclude that every simplex space contains largest $G M-, G M L C$-, and $G C$-ideals, and that both ideals and quotients of $G M-$, $G M L C$-, and $G C$-spaces are the same.

5. The main theorems. A subset of a topological space is $F_{\sigma}$ if it is a countable union of closed subsets of the space. We will show in $\$ \$ 7.2$ and 7.3 that neither condition (1) nor (2) of Theorem 5.1 may be omitted, even when $A$ is a separable $M$-space.

THEOREM 5.1. If $A$ is a separable simplex space, then $A$ is a GC-space if and only if

(1) $E P_{1}(A)$ is $F_{\sigma}$ in $P_{1}(A)$ (or equivalently is $K_{\sigma}$ ),

(2) closed subsets of $\max A$ are second category in themselves.

One may replace (2) by

(2') $\max A$ is strongly Baire.

Proof. Suppose that $A$ satisfies (1) and (2). We must show that each quotient $B$ of $A$ contains a nonzero $C$-ideal. Since the maps $\theta^{*}$ and $\theta^{-1}$ of (1.1) are homeomorphisms, we have that $E P_{1}(B)$ is $K_{\sigma}$ and $\max B$ is second category for each quotient $B$. Thus changing our notation, it suffices to show that if $A$ is a simplex space with $E P_{1}(A)$ a $K_{\sigma}$ set and $\max A$ second category, then $A$ contains a nonzero $C$-ideal.

Let $K_{n}$ be an increasing sequence of closed, and thus compact subsets of $P_{1}(A)$ with $E P_{1}(A)=\bigcup K_{n}$. We may assume that $0 \in K_{n}$ for all $n$. If $F$ is a closed subset of $K_{n}$ containing 0 , it is dilated in the simplex $P_{1}(A)$; hence its closed convex hull $Q=\bar{c}(F)$ is a face in $P_{1}(A)$ containing 0 [8, Theorem 3.3]. Since $F=E(Q)$ (see 
[14, p. 9]), $F-\{0\}$ is closed as a subset of $\max A$, and the weak* and structure topologies coincide on each set $K_{n}-\{0\}$. Since elements of $A$ are weak* continuous, they restrict to structurally continuous functions on $M_{A}\left(K_{n}-\{0\}\right)$. Since $\max A$ is second category, and is the union of the closed sets $M_{A}\left(K_{n}-\{0\}\right)$, one of the latter must have interior $G \neq \varnothing$ in $\max A$. Letting $J$ be the closed ideal in $A$ with $G=\max J$, elements of $A$ restrict to continuous functions on $\max J$, and from Theorem 2.2, $J \subseteq_{C} A$.

Conversely, suppose that $A$ is a $G C$-space. Since $A$ is separable, $P_{1}(A)$ is compact metric and thus separable. Let $J_{\gamma}, 0 \leqq \gamma \leqq \gamma_{0}$ be a $C$-composition series for $A$. The sets $J_{\gamma}^{1}$ form a strictly decreasing transfinite sequence of closed sets in $P_{1}(A)$; hence $\gamma_{0}$ must be countable (see $[11, \S 19$, II]). We have

$$
\max A=\bigcup_{y<\gamma_{0}}\left[\max J_{y+1}-\max J_{y}\right]
$$

hence

$$
\begin{aligned}
E P_{1}(A)-\{0\} & =\bigcup_{\gamma<\gamma_{0}}\left[\left(E P_{1}(A)-J_{y+1}^{\perp}\right)-\left(E P_{1}(A)-J_{y}^{\perp}\right)\right] \\
& =\bigcup_{\gamma<\gamma_{0}}\left[E\left(J_{y}^{\perp}\right)-E\left(J_{y+1}^{\perp}\right)\right] .
\end{aligned}
$$

It suffices to show that $B_{\gamma}=E\left(J_{y}^{1}\right)-E\left(J_{\gamma+1}^{1}\right)$ is $K_{\sigma}$. Let $\theta: A \rightarrow A / J_{\gamma}$ be the quotient map. From $\S 1, \theta^{*}: E P_{1}\left(A \mid J_{y}\right) \rightarrow E\left(J_{y}^{1}\right)$ is a homeomorphism; hence it suffices to show that

$$
B_{y}^{\prime}=\theta^{*-1}\left(B_{y}\right)=E P_{1}\left(A / J_{y}\right)-\left(J_{y+1} / J_{y}\right)^{1}
$$

is $K_{\sigma}$. Let $\rho_{1}: P_{1}\left(A / J_{\gamma}\right) \rightarrow P_{1}\left(J_{y+1} / J_{\gamma}\right)$ be the restriction map, and $\rho_{E}$ the restriction of $\rho_{1}$ :

$$
\rho_{E}: B_{\gamma}^{\prime}=E P_{1}\left(A / J_{y}\right)-\left(J_{y+1} / J_{y}\right)^{\perp} \rightarrow E P_{1}\left(J_{y+1} / J_{y}\right)-\{0\}
$$

(see (1.2)). $J_{y+1} / J_{y}$ is a $C$-space; hence $E P_{1}\left(J_{y+1} / J_{y}\right)$ is closed in $P_{1}\left(J_{y+1} / J_{y}\right)$ (see Proposition 2.1). It follows that $E P_{1}\left(J_{y+1} / J_{y}\right)-\{0\}$ is second countable, locally compact, and thus $K_{\sigma}$. From Lemma 1.1, $B_{\gamma}^{\prime}$ is also $K_{\sigma}$.

Since any $C$-ideal is an $M$-ideal with locally compact structure, $\max A$ is $G M L C$, and from Lemmas 3.4 and 3.5, it is strongly Baire.

THEOREM 5.2. If $A$ is a separable simplex space, then $A$ is a GMLC-space if and only if

(1) $R P_{1}(A)$ is $F_{\sigma}$ in $P_{1}(A)$ (or equivalently, is $K_{\sigma}$ ),

(2) closed subsets of $\max A$ are second category in themselves.

One may replace (2) by

$\left(2^{\prime}\right) \max A$ is strongly Baire.

Proof. Suppose that $A$ satisfies (1) and (2). From the same argument we used in Theorem 5.1, it suffices to show that if $A$ is a separable simplex space with $R P_{1}(A)$ a $K_{\sigma}$ set and $\max A$ second category, then $A$ contains a nonzero, $M L C$ ideal. 
Since $P_{1}(A)$ is metrizable and second countable, open subsets are $F_{\sigma}$, hence $R P_{1}(A)-\{0\}$ is $K_{\sigma}$. Let $K_{n}$ be compact sets with $R P_{1}(A)-\{0\}=\bigcup K_{n}$. If $F$ is a closed subset of $K_{n}$, let $D(F)$ be all $p \in P_{1}(A)$ such that for some $\alpha \geqq 0$ and $q \in F$, $p=\alpha q$. Then $D(F)$ is clearly dilated in $P_{1}(A)$, and we claim that it is closed. Suppose $p_{v}$ is a net in $D(F)$ converging to $p \in P_{1}(A)$. Let $p_{v}=\alpha_{v} q_{v}, q_{v} \in F, \alpha_{v} \geqq 0$. Choosing a subnet, we may assume that $q_{v} \rightarrow q \in F$. The sequence $\alpha_{v}$ must be eventually bounded, since if not, $q_{v} \in \alpha_{v}^{-1} P_{1}(A)$ will imply $q=0$. Choosing a subnet, we may assume $\alpha_{v} \rightarrow \alpha \geqq 0$. But then $\alpha_{v} q_{v} \rightarrow \alpha q, p=\alpha q$, and $p \in D(F)$. From [8, Theorem 3.3], $Q=\bar{c}(D(F))$ is a closed face in $P_{1}(A)$ with $E(Q) \subseteq D(F)$. We have (see (2.2))

$$
\bar{M}_{A}(F)=\bar{M}_{A}(D(F)-\{0\})=M_{A}(E(Q)-\{0\}) ;
$$

hence $\bar{M}_{A}(F)$ is closed in $\max A$. We conclude that $\bar{M}_{A}: K_{n} \rightarrow \max A$ is a closed map; hence, since $K_{n}$ is compact Hausdorff, $\bar{M}_{A}\left(K_{n}\right)$ is a compact Hausdorff space (see [3, p. 97]). But $\max A$ is second category and it is the union of the closed sets $\bar{M}_{A}\left(K_{n}\right)$; hence one of the latter has interior $G \neq \varnothing$ in $\max A . G$ is separated in $\bar{M}_{A}\left(K_{n}\right)$ and the latter is closed in $\max A$; hence $G$ is separated in $\max A$. It is also locally compact. Letting $J$ be the closed ideal in $A$ with $G=\max J$, we see that $J$ is an $M L C$-ideal in $A$.

Suppose that $A$ is a $G M$-space. $P_{1}(A)$ is compact metric, hence separable. Let $J_{\gamma}, 0 \leqq \gamma \leqq \gamma_{0}$, be an $M$-composition series for $A$. As in the proof of Theorem 5.1, $\gamma_{0}$ must be countable, and we have

$$
R P_{1}(A)-\{0\}=\bigcup_{\gamma<\gamma_{0}} R\left(J_{\gamma}^{\perp}\right)-R\left(J_{\gamma+1}^{\perp}\right)
$$

and it suffices to show that $B_{y}=R\left(J_{y}^{\perp}\right)-R\left(J_{y+1}^{\perp}\right)$ is $K_{\sigma}$. Let $\theta: A \rightarrow A / J_{y}$ be the quotient map.

$$
\theta^{*}: R P_{1}\left(A / J_{y}\right) \rightarrow R\left(J_{y}^{\perp}\right)
$$

is a homeomorphism; hence it suffices to show

$$
B_{y}^{\prime}=\theta^{*-1}\left(B_{y}\right)=R P_{1}\left(A / J_{y}\right)-\left(J_{y+1} / J_{y}\right)^{\perp}
$$

is $K_{\sigma}$. Let $\rho_{1}: P_{1}\left(A / J_{y}\right) \rightarrow P_{1}\left(J_{y+1} / J_{\gamma}\right)$ be the restriction map, and $\rho_{R}$ the restriction of $\rho_{1}$ :

$$
\rho_{R}: B_{y}^{\prime}=R P_{1}\left(A / J_{\gamma}\right)-\left(J_{y+1} / J_{y}\right)^{\perp} \rightarrow R P_{1}\left(J_{y+1} / J_{y}\right)-\{0\}
$$

(see (2.2)). Since $J_{y+1} / J_{y}$ is an $M$-space, $R P_{1}\left(J_{y+1} / J_{y}\right)$ is closed in $P_{1}\left(J_{y+1} / J_{y}\right)$. It follows that $R P_{1}\left(J_{\gamma+1} / J_{\gamma}\right)-\{0\}$ is second countable locally compact, and thus $K_{\sigma}$. Since $J_{\gamma+1} / J_{y}$ is an $M$-ideal in $A / J_{\gamma}$, we have from Lemma 2.7 that $B_{\gamma}^{\prime}$ is also $K_{\sigma}$.

If $A$ is $G M L C$, then from Lemmas 3.4 and $3.5, \max A$ is strongly Baire.

Note that we proved that if $A$ is separable, then $A$ is a $G M$-space implies $R P_{1}(A)$ is $K_{\sigma}$. By using the example in $[7, \S 5]$ one can easily show the converse is false.

THEOREM 5.3. If $A$ is a GM-space, then the following are equivalent:

(1) $\max A$ is almost locally compact Hausdorff (i.e., $A$ is a GMLC-space). 
(2) $\max A$ is strongly Baire.

(3) If $a \in A$, then for each closed subset $F$ of $\max A, a \mid F$ has a point of continuity in each subset of $F$ that is relatively open and separated in $F$.

Proof. (1) $\Rightarrow(2)$. This follows from Lemmas 3.4 and 3.5.

(2) $\Rightarrow$ (3). If $I$ is a proper closed ideal in $A$ with $F=\max A / I$, we may identify the functions $a \mid F$ with the elements of $A / I$. In addition, closed subsets of $\max A$ are Baire. Thus it suffices to show that if $A$ is a simplex space with Baire structure space, then each $a \in A$ has a point of continuity in each nonempty separated open subset $G$. Since $G$ is second category, it will suffice to show that $f=a \mid G$ is of first Baire class, i.e., if $S$ is a closed subset of 'he reals, then $f^{-1}(S)$ is a $G_{\delta}$ subset of $G$ (see $[11, \S 27, \mathrm{X}]$ ). The difference of two first Baire class functions is again the same (see $[11, \S 27, \mathrm{VI}]$ ) and $A=A^{+}-A^{+}$; hence we may assume $a \geqq 0$.

It will suffice to show that $f$ is upper semicontinuous on $G$, i.e., given $\alpha>0$, the set

$$
F=\{M \in G: f(M) \geqq \alpha\}=G \cap\{M \in \max A: a(M) \geqq \alpha\}
$$

is relatively closed in $G$. Suppose that $M_{\nu} \in F$ converges to $M \in G$. Since $C \equiv$ $\{M \in \max A: a(M) \geqq \alpha\}$ is compact [7, Proposition 4.5], taking a subnet we may assume that $M_{v}$ converges to $N \in C$. Since $G$ is separated in $\max A, M=N$ and $M \in F$.

(3) $\Rightarrow$ (1). It suffices to show that if $A$ is $G M$ and each $a \in A$ has a point of continuity in each nonempty open separated subset, then $\max A$ contains a nonempty locally compact separated open subset. Since $A$ is $G M$, there is a separated open subset $G$ in $\max A$. Let $G=\max J, J$ a closed ideal in $A$, and choose $a \in J^{+}$, $a \neq 0$. The set

$$
G_{1}=\{M \in \max A: a(M)>0\}
$$

is an open subset of $G$ [7, Proposition 4.7], and thus is separated in max $A$. Let $M_{0} \in G_{1}$ be a point of continuity of $a$. Then letting $a\left(M_{0}\right)=\varepsilon>0$, the set

$$
C=\{M \in \max A: a(M) \geqq \varepsilon / 2\}
$$

must have nonempty interior $G_{2}$ with $M_{0} \in G_{2}$. On the other hand $G_{2}$ is an open subset of the compact Hausdorff space $C$, and thus is locally compact. $G_{2}$ is separated in $\max A$ since $G_{2} \subseteq G$.

COROLlARY 5.4. If $A$ is an $M$-space, then the following are equivalent:

(1) $\max A$ is almost locally compact.

(2) $\max A$ is strongly Baire.

(3) If $a \in A$, then for each closed subset $F$ of $\max A$, the points of continuity of a $F$ are dense in $F$.

It might at first seem rather difficult to determine whether $\operatorname{EP}_{1}(A)$ or $R P_{1}(A)$ are $F_{\sigma}$ in $P_{1}(A)$. That this is often not the case is due to a result in point set topology. 
If $S$ is a subset of a topological space $X$, define the residue $\rho(S)$ of $S$ in $X$ by $\rho(S)=[\bar{S}-S]^{-} \cap S$. We have (see $\left.[11, \S 30, \mathrm{VI}]\right)$ :

Proposition 5.5. If $X$ is a complete metric space, then a subset $S$ of $X$ is both $G_{\delta}$ and $F_{\sigma}$ if and only if for each nonempty relatively closed subset $F$ of $S, \rho(F)$ is a proper subset of $F$.

If $A$ is separable, this is applicable to $E P_{1}(A)$, since then $P_{1}(A)$ is metrizable, and $E P_{1}(A)$ is already $G_{\delta}$ (see [14, Proposition 1.3]). The same is true for $R P_{1}(A)$ since

LemMA 5.6. If $A$ is a separable simplex space, then $R P_{1}(A)$ is $G_{\delta}$ in $P_{1}(A)$.

Proof. We say that $q, r \in P_{1}(A)$ are proportional if there is an $\alpha \in[0,1]$ with $q=\alpha r$ or $r=\alpha q$. We have $p \notin R P_{1}(A)$ if and only if there exist nonproportional $q, r \in P_{1}(A)$ such that $p=\frac{1}{2} q+\frac{1}{2} r$. To show this, note $p \notin R P_{1}(A)$ if and only if $p \neq 0$ and

$$
p /\|p\| \notin E P_{1}(A)-\{0\}=E S(A) .
$$

But the latter will be true if and only if $p /\|p\|$ is not the midpoint of two distinct elements of $S(A)$.

Define $F_{1}, F_{2} \subseteq P_{1}(A) \times P_{1}(A)$ by

$$
\begin{aligned}
& F_{1}=\{(q, r): q=\alpha r, \alpha \in[0,1]\}, \\
& F_{2}=\{(q, r): \alpha q=r, \alpha \in[0,1]\},
\end{aligned}
$$

and $\psi: P_{1}(A) \times P_{1}(A) \rightarrow P_{1}(A)$ by

$$
\psi((p, q))=\frac{1}{2} p+\frac{1}{2} q .
$$

We have $R P_{1}(A)=P_{1}(A)-\psi(G)$, where $G=P_{1}(A) \times P_{1}(A)-F_{1} \cup F_{2}$. Since the sets $F_{i}$ are closed, $G$ is open in the compact metric space $P_{1}(A) \times P_{1}(A)$, and thus is $K_{\sigma}$. Since $\psi$ is continuous, $\psi(G)$ is $K_{\sigma}$, and $R P_{1}(A)$ is $G_{\delta}$.

We note that if $E P_{1}(A)$ is $K_{\sigma}$, then so is $R P_{1}(A)=[0,1] E P_{1}(A)$, since products of compact sets are the same. The converse is false (see $§ 7.2$ ).

For a typical application of Proposition 5.5 , let $P_{\alpha}(A), \alpha \geqq 0$, be the positive functions $p$ satisfying $\|p\| \leqq \alpha$.

Proposition 5.7. Let $A$ be a separable simplex space. If for some $\alpha<1$ we have

$$
Z_{A}=\left[E P_{1}(A)\right]^{-} \subseteq E P_{1}(A) \cup P_{\alpha}(A),
$$

then $E P_{1}(A)$ is $F_{\sigma}$ in $P_{1}(A)$.

Proof. Since $P_{\alpha}(A)$ is closed,

$$
\rho\left(E P_{1}(A)\right)=\left[Z_{A}-E P_{1}(A)\right]^{-} \cap E P_{1}(A) \subseteq P_{\alpha}(A) \cap E P_{1}(A)=\{0\} ;
$$

hence $\rho\left(\rho\left(E P_{1}(A)\right)\right)=\rho(\{0\})=\varnothing$. It follows that $\rho$ must map nonempty relatively closed subsets of $E P_{1}(A)$ onto proper subsets. 
It is interesting to note that if $X$ is locally compact Hausdorff (e.g., if $X=P_{1}(A)$ ), then a subset $S$ of $X$ is itself locally compact if and only if it is locally closed, or equivalently, $\rho(S)=\varnothing$ (see $[12, \S 39, \mathrm{VII}])$.

6. The Choquet boundary. We wish to prove an analogue of the Bishop-de Leeuw characterization of the Choquet boundary of a function algebra (see [14, $\S 8])$. Suppose that $X$ is a compact Hausdorff space and that $A$ is a linear subspace of $C(X)$ that is a simplex space with the relative norm and ordering. If $x \in X$, the functional $\delta(x)$ defined on $A$ by $\delta(x)(a)=a(x)$ is positive and of norm less than or equal to 1 , i.e., $\delta(x) \in P_{1}(A)$. We define the Choquet boundary $\partial A$ to be the points $x \in X$ such that $\delta(x) \in E P_{1}(A)$. We say $x \in X$ is a semipeak point if $\delta(x)=0$ or for each $y \in X$ with $\delta(y) \neq \delta(x)$, there is an $a \in A$ with

$$
a(y)<a(x)=\|a\| \text {. }
$$

THEOREM 6.1. The Choquet boundary $\partial A$ consists of the semipeak points, and $\delta(\partial A)$ equals either $E P_{1}(A)-\{0\}$ or $E P_{1}(A)$.

Proof. The argument given in [8, Lemma 4.1] is still valid; hence $\delta(X) \supseteq E P_{1}(A)$ $-\{0\}$, and the second assertion follows.

If $\delta(x) \in E P_{1}(A)-\{0\}$, and $\delta(y) \neq \delta(x)$, let $Q$ be the smallest closed face in $P_{1}(A)$ containing $\delta(y)$. If $Q$ is contained in the line segment $[0, \delta(x)]$ joining 0 and $\delta(x)$, then there is an $\alpha$ with $0 \leqq \alpha<1$ and $\delta(y)=\alpha \delta(x)$. If $a \in A$ and $a(x)>0$,

$$
a(y)=\alpha a(x)<a(x),
$$

and to prove (6.1) it suffices to select $a$ with $a(\delta(x))=\|a\|>0$. The existence of such an element was proved in [8, Lemma 4.1]. If $Q \nsubseteq[0, \delta(x)]$, choose $q \in E(Q)$ $-\{0, \delta(x)\} . M_{q}=[0, q]^{\perp}$ is a (maximal) closed ideal in $A[7$, Corollary 3.2], and from the proof of [7, Theorem 4.4], if $\rho_{1}$ is the restriction map,

$$
\rho_{1}\left(E P_{1}(A)-\{0, q\}\right)=E P_{1}\left(M_{q}\right)-\{0\} \text {. }
$$

The previously mentioned existence theorem gives us an element $a \in M_{q}$ with $a(p)=\|a\| \neq 0$. The set

$$
R=\left\{r \in P_{1}(A): a(r)=\|a\|\right\}
$$

is a closed face in $P_{1}(A)$. If $a(\delta(y))=a(y)=\|a\|$, then $\delta(y) \in R, Q \subseteq R$, and $q \in R$. This contradicts the fact that $a(q)=0$. Thus $x$ is a semipeak point.

Conversely, suppose that $\delta(x) \notin E P_{1}(A)$, and let $Q$ be the smallest closed face in $P_{1}(A)$ containing $\delta(x)$. Since $E(Q) \subseteq E P_{1}(A)$ we may choose $y \in X$ with $\delta(y) \in E(Q)$ $-\{0\}$. We have $\delta(y) \neq \delta(x)$. If $a \in A$, define $R$ as above. Then if $a(x)=\|a\|, \delta(x) \in R$ and $Q \subseteq R$. It follows that $\delta(y) \in R$, i.e., $a(y)=\|a\|$, and inequality (6.1) cannot hold. Thus $x$ is not a semipeak point.

In the following section, we will say that $x \in X$ is a peak point if there is an $a \in A$ such that $0 \leqq a(y)<a(x)$ for all $y$ with $\delta(y) \neq \delta(x)$. It is clear that a peak point must be a semipeak point. 


\section{Some examples.}

7.1. A closed ideal which is an $M$-space but not an $M$-ideal. Let $J$ be the positive integers, and $J^{*}=J \cup\{\infty\}$ the one-point compactification of $J$. Let $A \subseteq C\left(J^{*}\right)$ be all functions $a$ such that

$$
a(\infty)=\frac{1}{2} a(1)+\frac{1}{2} a(2),
$$

together with the relative norm and ordering. $A$ is a simplex space. (See $[2$, Theorem 1], $[13$, p. 78]. The proof simply uses the fact that the map $\rho$ defined as in $[8$, Lemma 4.1] is one-to-one and onto $S(A)$.) Define $\varepsilon_{n}$ on $J^{*}$ for $1 \leqq n \leqq \infty$ by

$$
\begin{aligned}
\varepsilon_{n}(m) & =0 & & m \neq n \\
& =1 & & m=n .
\end{aligned}
$$

Then $\varepsilon_{n}$ is in $A$ for $3 \leqq n<\infty$, as are the functions

$$
f_{1}=2 \varepsilon_{1}+\sum_{3 \leqq i \leqq \infty} \varepsilon_{i}, \quad f_{2}=2 \varepsilon_{2}+\sum_{3 \leqq i \leqq \infty} \varepsilon_{i} .
$$

Noting that in particular $f_{1}$ assumes distinct values at 1,2 , and $\infty$, we have that $A$ distinguishes points in $J^{*}$, hence $\delta: J^{*} \rightarrow P_{1}(A)$ is a homeomorphism. Using $\varepsilon_{n}$, $3 \leqq n<\infty$, it is clear that $n$ is a peak point for these values. 1 is a peak point since $f_{1}(1)>f_{1}(n)$ for all $n \neq 1$. Similarly 2 is a peak point. $\infty$ is not a peak point since from (7.1), $a(\infty)$ must lie between $a(1)$ and $a(2)$. Thus $\partial A=J$, and from Theorem 6.1 ,

$$
E P_{1}(A)=\{0\} \cup\{\delta(n): 1 \leqq n<\infty\} .
$$

From [7, Corollary 3.2],

$$
I=\{a \in A: a(1)=0\}=\{\delta(1)\}^{\perp}
$$

is a (maximal) closed ideal in $A$ since the line segment joining 0 and $\delta(1)$ is a closed face in $P_{1}(A) . I$ may be identified with

$$
\left\{a \in C\left(J^{*}-\{1\}\right): a(\infty)=\frac{1}{2} a(2)\right\} \text {. }
$$

Since the latter is a sublattice of $C\left(J^{*}-\{1\}\right)$, it is an $M$-space. On the otherhand, $n \rightarrow \infty$, hence

$$
\delta(n) \rightarrow \delta(\infty)=\frac{\delta(1)+\delta(2)}{2}
$$

and from Lemma 2.3, $M_{\delta(n)}$ converges to both $M_{\delta(1)}$ and $M_{\delta(2)}$. It follows that although $M_{\delta(2)} \in \max I$, it is not a separated point in $\max A$.

Although $I$ is not a $C$-space, $A$ and thus $I$ are $G C$-spaces. Letting

$$
I_{1}=\left\{a \in C\left(J^{*}\right): a(1)=a(2)=0\right\},
$$

we have that $\{0\} \subseteq I_{1} \subseteq A$ is a $C$-composition series since $I_{1}$ may be identified with $C_{0}\left(J^{*}-\{1,2, \infty\}\right)$, and $A / I_{1}$ with $C(\{1,2\})$. 
7.2. A locally compact $M$ (i.e., $M L C$ ) space which is nowhere $C$ (i.e., an NCspace). We will construct a separable $M$-space $A$ for which max $A$ is compact, and

$$
E P_{1}(A)-\{0\} \subseteq\left[E P_{1}(A)^{-}-E P_{1}(A)\right]^{-} .
$$

If $J$ is a $C$-ideal in $A$, we have from Theorem 2.2, $E P_{1}(A)^{-} \subseteq E P_{1}(A) \cup J^{\perp}$; hence

$$
E P_{1}(A) \subseteq\left[E P_{1}(A)^{-}-E P_{1}(A)\right]^{-} \cup\{0\} \subseteq J^{\perp},
$$

and $J^{\perp}=P_{1}(A)$, i.e., $J=\{0\}$.

Let $C_{b}([0, \infty))$ be the bounded continuous real functions on $[0, \infty)$ with the uniform norm and the usual ordering. $C_{b}([0, \infty))$ may be identified with $C(\beta[0, \infty))$ where $\beta[0, \infty)$ is the Stone-Čech compactification of $[0, \infty)$, since each $f \in C_{b}([0, \infty))$ has an isometric order isomorphic extension to $\beta[0, \infty)$. Thus $C_{b}([0, \infty))$ is a $C$ space.

Let $\alpha_{n}, n \geqq 1$ be a sequence of scalars with $0<\alpha_{n}<1$, and $\prod \alpha_{n}>0$ (e.g., let $\alpha_{n}=1$ $-1 /(n+1)^{2}$-see [1, p. 155]). Let $r_{n}, n \geqq 1$ be an enumeration of the rationals in $[0, \infty)$. We may assume that $r_{n} \leqq n-1$, hence in particular, $r_{1}=0$. We define $A_{0}$ to be the functions $a$ in $C_{b}([0, \infty))$ such that $a(n)=\alpha_{n} f\left(r_{n}\right), n \geqq 1$. Although it can be shown that $A_{0}$ has the desired properties, it is not separable. We will use instead the sublattice generated by a countable subset.

Define $a_{0} \in C_{b}([0, \infty))$ inductively: Let $a_{0}(0)=1$. Having defined $a_{0}(x)$ for $0 \leqq x \leqq n$, and using the fact that $r_{n+1} \leqq n$, let

$$
a_{0}(n+1)=\alpha_{n+1} a_{0}\left(r_{n+1}\right),
$$

and interpolate linearly between $n$ and $n+1$. It is clear that $a_{0} \in A_{0}$, and that $a_{0}$ is strictly decreasing. In particular $a_{0}$ distinguishes points in $[0, \infty)$. We' also have that if $0<x \leqq n$,

$$
a_{0}(x) \geqq a_{0}(n)=\alpha_{n} a_{0}\left(r_{n}\right) \geqq \alpha_{n} a_{0}(n-1) ;
$$

hence, letting $\varepsilon=\prod \alpha_{n}$,

$$
a_{0} \geqq \varepsilon 1 .
$$

To obtain peaking at points other than the integers, we define $a_{k} \in C_{b}([0, \infty))$, $k \geqq 1$, inductively. Let $a_{k}(0)=1$. Having defined $a_{k}(x)$ for $0 \leqq x \leqq n$, define $a_{k}(n+1)$ as in (7.3). Let $a_{k}(x)=1$ for $x \in[n+1 / 2 k, n+1-1 / 2 k]$, and interpolate linearly in the intervals $[n, n+1 / 2 k]$ and $[n+1-1 / 2 k, n+1]$.

We let $A$ be the smallest closed lattice subspace containing $a_{k}, 0 \leqq k<\infty$. To see that $A$ is separable, let $B$ be all finite linear combinations $\sum \alpha_{i} a_{i}$ with $\alpha_{i}$ nonnegative rationals. $B$ is closed under addition and multiplication by nonnegative rationals. Let $C$ be all elements of the form $b_{1} \vee \cdots \vee b_{m}$ with $b_{i} \in B$. $C$ is closed under unions, additions, and multiplication by nonnegative rationals, since

$$
\left(b_{1} \vee \cdots \vee b_{m}\right)+\left(b_{1}^{\prime} \vee \cdots \vee b_{n}^{\prime}\right)=\bigvee_{i, j}\left(b_{i}+b_{j}^{\prime}\right)
$$


and

$$
\alpha\left(b_{1} \vee \cdots \vee b_{m}\right)=\alpha b_{1} \vee \cdots \vee \alpha b_{m} .
$$

$C-C$ is closed under unions since

$$
\left(c_{1}-c_{2}\right) \vee\left(c_{3}-c_{4}\right)=\left(c_{1}+c_{4}\right) \vee\left(c_{3}+c_{2}\right)-\left(c_{2}+c_{4}\right),
$$

and $C-C$ is closed under addition and multiplication by all rational scalars. Since these three operations are continuous in $C_{b}([0, \infty)$ ) (see [5, p. 99] for unions), the closure $[C-C]^{-}$is again closed with respect to them. It follows that $[C-C]^{-}$is closed with respect to multiplication by all scalars, and is a closed vector lattice. Thus $A=[C-C]^{-}$, and since $C-C$ is countable, $A$ is separable.

We will regard $A$ as a sublattice of $C(\beta[0, \infty))$. Since $A \subseteq A_{0}$,

$$
\delta(n)=\alpha_{n} \delta\left(r_{n}\right), \quad \alpha_{n}<1, n \geqq 1 ;
$$

hence $\|\delta(n)\|<1$, and, letting $\boldsymbol{J}$ be the positive integers,

$$
\delta(J) \cap E P_{1}(A)-\{0\}=\varnothing .
$$

If $x \in[0, \infty)-J$, then choosing integers $n \geqq 0, k \geqq 1$ with

$$
n+1 / 2 k \leqq x \leqq n+1-1 / 2 k,
$$

we have that $a_{k}(x)=1,\|\delta(x)\|=1$, and $\delta(x) \in E P_{1}(A)$; $(\delta(x)$ already lies on an extremal ray). Since $[0, \infty)-J$ is dense in $[0, \infty)$, we have

$$
\delta(J) \subseteq E P_{1}(A)^{-}-E P_{1}(A) .
$$

To prove (7.2), note that $\delta(J)^{-} \supseteq \delta([0, \infty))$, since letting $x \in[0, \infty)$, and $r_{n_{k}}$ be a subsequence of $r_{n}$ with $r_{n_{k}} \rightarrow x$, we have $\alpha_{n_{k}} \rightarrow 1$ and, from (7.5),

$$
\delta\left(n_{k}\right)=\alpha_{n_{k}} \delta\left(r_{n_{k}}\right) \rightarrow \delta(x) .
$$

$[0, \infty)$ is dense in $\beta[0, \infty)$; hence

$$
\delta(J)^{-} \supseteq \delta(\beta[0, \infty)) \supseteq E P_{1}(A)-\{0\},
$$

the latter inclusion being a consequence of Theorem 6.1.

Finally, since $a_{0}$ must vanish at $\infty$ [7, Proposition 4.5], we have from (7.4) that $\max A$ is compact Hausdorff, hence strongly Baire. From (7.4), $F=E P_{1}(A)-\{0\}$ is relatively closed in $E P_{1}(A)$. Taking residues (see $\left.§ 5\right)$, a simple argument shows that $\rho(F)=\rho\left(E P_{1}(A)\right)$. From (7.2), $\rho(F)=F$. Using Proposition 5.5 we conclude that $E P_{1}(A)$ is not $K_{\sigma}$. Thus $A$ satisfies (2) but not (1) of Theorem 5.1.

7.3. An M-space which is nowhere locally compact (i.e., an NMLC-space). We will construct a separable $M$-space $A$ such that $\max A$ is countable and dense in itself, i.e., if $M \in \max A$, then $M \in[\max A-\{M\}]^{-}$. If $G$ were a nonempty locally compact open subset of $\max A, G$ would also be dense in itself. But $G$ would be second category, and letting $G=\left\{M_{1}, M_{2}, \ldots\right\}, G-\left\{M_{i}\right\}$ would be dense in $G$; hence $\varnothing=\bigcap_{i} G-\left\{M_{i}\right\}$ would be the same, a contradiction. 
Let $J^{*}=J \cup\{\infty\}$ be the one-point compactification of the positive integers. Let $\varphi$ be a map of $J-\{1\}$ onto $J \times J$ such that if $\varphi(m)=(p, q)$, then $p<m$. Let $\alpha_{m}, m \geqq 2$ be any sequence with $0<\alpha_{m}<1$ and $\alpha_{m}$ decreasing to zero. We define $A$ to be the functions $a \in C\left(\mathrm{~J}^{*} \times \mathrm{J}^{*}\right)$ such that

$$
\begin{aligned}
a(1, \infty) & =0, & & \\
a(m, \infty) & =\alpha_{m} a(\varphi(m)), & & 2 \leqq m \leqq \infty . \\
a(\infty, n) & =0, & & 1 \leqq n \leqq \infty .
\end{aligned}
$$

If $1 \leqq i, j<\infty$, define a function $a_{i j}$ on $J^{*} \times J^{*}$ as follows:

$$
\begin{aligned}
a_{i j}(m, n) & =0, \quad m<i, 1 \leqq n \leqq \infty, \\
a_{i j}(i, n) & =0, \quad n \neq j, 1 \leqq n \leqq \infty, \\
a_{i j}(i, j) & =1 .
\end{aligned}
$$

For $m>i$, we proceed inductively. Suppose that $a_{i j}(m, n)$ has been defined for $i \leqq m \leqq m_{0}$, and $1 \leqq n \leqq \infty$. Then define

$$
a_{i j}\left(m_{0}+1, n\right)=\alpha_{m_{0}+1} a_{i j}\left(\varphi\left(m_{0}+1\right)\right), \quad 1 \leqq n \leqq \infty .
$$

Note that if $\varphi\left(m_{0}+1\right)=(p, q)$, then $p \leqq m_{0}$, and $a_{i j}\left(\varphi\left(m_{0}+1\right)\right)$ is already defined. Finally define

$$
a_{i j}(\infty, n)=0, \quad 1 \leqq n \leqq \infty
$$

We claim that $a_{i j}$ is continuous. We have

$$
\begin{array}{ll}
a_{i j}(m, \infty)=\lim _{n} a_{i j}(m, n)=0, & 1 \leqq m \leqq i \\
a_{i j}(m, \infty)=\lim _{n} a_{i j}(m, n)=\alpha_{m} a_{i j}(\varphi(m)), & i<m<\infty
\end{array}
$$

On the other hand, if $i<m<\infty$,

$$
a_{i j}(m, n) \leqq \alpha_{m}, \quad 1 \leqq n \leqq \infty .
$$

If $\left(m_{k}, n_{k}\right)$ is a sequence converging to $(\infty, n)$, then for any $m_{0}>i$, eventually $m_{k} \geqq m_{0}$, and $a_{i j}\left(m_{k}, n_{k}\right) \leqq \alpha_{m_{0}}$; hence

$$
\lim _{k} a_{i j}\left(m_{k}, n_{k}\right)=a_{i j}(\infty, n)=0 .
$$

It is clear that $a_{i j}$ satisfies (7.6) and thus is an element of $A$.

Since $a_{i j}(i, j)=1$ and $a_{i j}(m, n)<1$ for $(m, n) \neq(i, j)$, we have that $J \times J \subseteq \partial A$, and $A$ distinguishes points in $J \times J$. From (7.6),

$$
\begin{aligned}
\delta((1, \infty)) & =0, & & \\
\delta((m, \infty)) & =\alpha_{m} \delta(\varphi(m)), & & 2 \leqq m<\infty \\
\delta((\infty, n)) & =0, & & 1 \leqq n \leqq \infty
\end{aligned}
$$


hence $\delta(J \times J)=E P_{1}(A)-\{0\}$. Given any pair $(p, q)$, letting $\varphi(m)=(p, q)$ we have $(m, n) \underset{n}{\rightarrow}(m, \infty)$; hence

$$
\delta((m, n))_{n} \delta((m, \infty))=\alpha_{m} \delta((p, q))
$$

From Lemma 2.3,

$$
M_{\delta((m, n))} \vec{n} M_{\delta((p, q))}
$$

and $\max A$ is dense in itself.

Since $E P_{1}(A)-\{0\}=\delta(J \times J)$ is countable, it is $K_{\sigma}$, and $A$ satisfies (1) but not (2) of Theorem 5.1. We note that as a consequence of [8, Corollary 3.9], $\max A$ is not first countable, even though it is a countable set.

\title{
REFERENCES
}

1. L. Ahlfors, Complex analysis, McGraw-Hill, New York, 1953.

2. E. Alfsen, On the geometry of Choquet simplexes, Math. Scand. 15 (1964), 97-110.

3. N. Bourbaki, Topologie générale, Chapters 1-2, Hermann, Paris, 1951.

4. E. Davies, The structure and ideal theory of the pre-dual of a Banach lattice, Trans. Amer. Math. Soc. 131 (1968), 544-555.

5. M. Day, Normed linear spaces, Academic Press, New York, 1962.

6. J. Dixmier, Les $C^{*}$-algebres et leurs applications, Gauthier-Villars, Paris, 1964.

7. E. Effros, Structure in simplexes, Acta Math. 117 (1967), 103-121.

8. —_, Structure in simplexes. II, J. Functional Analysis 1 (1967), 379-391.

9. I. Kaplansky, The structure of certain operator algebras, Trans. Amer. Math. Soc. 70 1951), 219-255.

10. J. Kelley, General topology, Van Nostrand, Princeton, N. J., 1955.

11. C. Kuratowski, Topologie. I, Mongr. Mat. 20, PWN, Warsaw, 1952.

12. - Topologie. II, Mongr. Mat. 21, PWN, Warsaw, 1950.

13. J. Lindenstrauss, Extensions of compact operators, Mem. Amer. Math. Soc. No. 48, 1964.

14. R. Phelps, Lectures on Choquet's theorems, Van Nostrand, New York, 1966.

15. M. Rogalski, Espaces de Banach ordonnés, simplexes, frontières de Šilov et problème de Dirichlet, C. R. Acad. Sci. Paris 263 (1966), 726-729.

16. H. Bauer, Silovscher Rand und Dirichletsches Problem, Ann. Inst. Fourier (Grenoble) 11 (1961), 89-136.

\author{
University of Pennsylvania, \\ Philladelphia, Pennsylvania \\ Stanford UNIVERSITY, \\ Stanford, California
}

